\title{
Development of a Wearable Gait Monitoring System for Evaluating Efficacy of Walking Training in Rehabilitation
}

\author{
Kosuke Motoi ${ }^{1, * * *}$, Sayaka Taniguchi ${ }^{1,2}$, Mina Baek $^{1}$, Morikuni Wakugawa ${ }^{2}$, \\ Takumi Sonoda ${ }^{2}$, Tadahiko Yuji ${ }^{1,2}$, Yuji Higashi ${ }^{2}$, Toshiro Fujimoto ${ }^{2}$, \\ Mitsuhiro Ogawa ${ }^{1}$, Shinobu Tanaka ${ }^{1}$ and Ken-ichi Yamakoshi ${ }^{1}$ \\ ${ }^{1}$ College of Science and Engineering, Kanazawa University, \\ Kakuma-machi, Kanazawa, Ishikawa 920-1192, Japan \\ ${ }^{2}$ Fujimoto Hayasuzu Hospital, \\ 17-1 Hayasuzu-machi, Miyakonojo, Miyazaki 885-0055, Japan
}

(Received October 4, 2011; accepted February 16, 2012)

Key words: wearable, posture change, walking speed, accelerometer, gyrosensor, rehabilitation

To evaluate the efficacy of walking training in rehabilitation, physical therapists need to assess motion characteristics. However, they often evaluate these activities subjectively by direct observation. In this study, we have developed a wearable system for monitoring posture changes and walking speed in patients with hemiplegia. The system can be used to measure the angle changes relative to the sagittal plane of the trunk, thigh, and shank, and walking speed using the angle changes of the lower limb and leg length. In 12 healthy subjects (from 21 to 75 years) and 10 patients with hemiplegia (from 50 to 87 years), we evaluated the measurement accuracy of the system by simultaneous recordings using charge-coupled device (CCD) cameras. Also, in four patients with hemiplegia, the gait motions were monitored during the rehabilitation to evaluate its applicability to the quantitative determination of the efficacy of the walking training. The results demonstrated that the system could be used to measure the angle changes of the body parts $(r=0.99)$ and walking speed (healthy subjects: $r=0.99$, patients: $r=0.95$ ) with high accuracy. It is also clearly shown through practical monitoring that the system can be a useful means of evaluating the motion characteristics during the rehabilitation of the patients, therefore, the efficacy of the rehabilitation can be quantitatively evaluated using the system.

${ }^{*}$ Corresponding author: e-mail: motoi@cc.hirosaki-u.ac.jp

${ }^{* *}$ Present affiliation: Graduate School of Science and Technology, Hirosaki University 


\section{Introduction}

The importance of activity monitoring is well recognized in the fields of rehabilitation, gerontology, and healthcare. For example, in the field of gerontology, it is necessary for the elderly to actively maintain their daily living to avoid being bedridden. Therefore, the quantitative measurement of these activities is essential. ${ }^{(1)}$

To evaluate the efficacy of a rehabilitation program, a physical therapist (PT) must assess the walking speed and posture changes during walking, standing, standingup, sitting, and so on. However, the PT must usually assess subjectively by direct observation. One method used is to make recordings using a motion capture system with charge-coupled device (CCD) cameras and a force plate. However, the range over which such recording is possible is usually limited and data analysis is complicated. Therefore, this method is generally not practical in rehabilitation.

For quantitative assessment, some wearable systems for monitoring the activities using an accelerometer, a gyrosensor, a pressure sensor, and so on, have been developed. ${ }^{(2-18)}$ For example, a wristwatch-type accelerometer was frequently used for the analysis of activity. ${ }^{(2,3)}$ However, the information obtained using this system showed only whether the subject was moving or resting. The systems discriminating whether the subject was standing, sitting, lying, walking, and so on, were also reported.(4-9) However, detailed motion characteristics during dynamic posture such as walking could not be obtained by these methods. Also, by using the methods in which the accelerometer was attached to the subject's trunk, only balance during standing or walking could be assessed. ${ }^{(10,11)}$ A device that assesses walking by measuring plantar pressure cannot continuously analyze movement of the body segments. ${ }^{(12)}$ These devices are available for the rough estimation of physical activity or gait pattern. However, more detailed information is usually required, particularly in the evaluation of the walking training during the rehabilitation.

Also, the methods of detecting angular changes in the lower limbs or walking speed using gyrosensors were reported. ${ }^{(13-15)}$ However, these systems could not be used to obtain the initial angle for integrating angular velocity from a gyrosensor and also for compensating the data drift. To solve these problems, joint angle measurements of the lower limb using an accelerometer together with a gyroscope were reported. ${ }^{(16-18)}$ However, they must require complex calculation and could not simultaneously measure the angle changes of the trunk, thigh, shank, and knee joint, and the walking speed that the PT needs to evaluate during walking training. Therefore, the applicability of these methods in the rehabilitation may not be suitable.

On the other hand, we reported the different concepts for monitoring activities. The measurement principle was quite simple. If we can measure the angle of three anatomical parts, such as the trunk, thigh, and shank, with respect to the gravitational direction, we can discriminate almost all the human postures in the sagittal plane, which are possible under normal daily life. ${ }^{(19)}$ Thus, we developed a portable device for measuring the trunk, thigh, and shank angles relative to the gravitational direction to improve the quality of life of elderly persons. At the start of the development, we used miniature electromagnetic inclinometers to measure angles, ${ }^{(19)}$ but had problems with the limited measurement range and inertial artifacts of the weight built in the 
sensor. To solve these problems, we used accelerometers and gyrosensors for the angle measurement and developed our system to measure not only the posture changes, but also the walking speed using the thigh angle changes. ${ }^{(20,21)}$

Using our concept for the activity monitoring, the motion characteristics of the patients under the rehabilitation could be clearly determined through practical measurement during rehabilitation. ${ }^{(22)}$ However, in the previous system that we developed, we used a compass model based on only angle changes in the thigh for walking speed measurement; therefore, the measurement precision was insufficient to evaluate the gait of a stroke patient during the rehabilitation. The analytical method and parameter for detecting the motion characteristics during walking training were not investigated. These drawbacks obstruct its practical use for the patient's gait monitoring in the rehabilitation field.

From these viewpoints, the objective of this study is to develop a wearable gait monitoring system to evaluate the efficacy of walking training in rehabilitation by improving the previous monitoring system mentioned above. To resolve these practical drawbacks mentioned earlier for the rehabilitation, we have newly designed a method of monitoring walking speed and analyzing the motion characteristics of a stroke patient during walking training. Then, by combining these new methods, we developed a new wearable device for monitoring posture changes and walking speed for use in the walking training during the rehabilitation. We have also investigated its applicability to the rehabilitation of four patients with hemiplegia.

\section{Materials and Methods}

Figure 1 shows the outline of the wearable gait monitoring system for use in the evaluation of motion during walking training in the rehabilitation room developed in this study. The three sensor units $\left(42 \times 60 \times 15 \mathrm{~mm}^{3}, 40 \mathrm{~g}\right)$ are attached to the patient's trunk, thigh, and shank.

The accelerometer (H34C, Hitachi metals, 3 axes, $\pm 2 \mathrm{G}$ ), gyrosensor (ENC-03R, Murata, 1 axis, $\pm 300 \mathrm{deg} / \mathrm{s}$ ), amplifier, micro SD card, transmitter (ZigBee, $2.4 \mathrm{GHz}$ ), battery $(3.7 \mathrm{~V}, 500 \mathrm{mAh})$, and CPU (10 bit A/D converter, sampling frequency of $25 \mathrm{~Hz})$ are installed in the sensor unit. The sampling frequency of $25 \mathrm{~Hz}$ was determined on the basis of the results of the practical monitoring of the patients in a previous study ${ }^{(22)}$ and thus is suitable for the gait monitoring. With the sensor system and personal computer, the patient's motion in the rehabilitation room can be monitored. The wireless system enables continuous monitoring for $6 \mathrm{~h}$. The data is transferred to the computer in real time and also stored on an SD card as back-up data.

Firstly, the angles of the trunk, thigh, and shank relative to the gravitational direction are calculated from the gravity variation component $(\mathrm{DC} \sim 0.5 \mathrm{~Hz})$ of the accelerometers. By using these angle values, it discriminates among the postures such as walking, standing, sitting, and lying. ${ }^{20,21)}$ In the static posture such as standing, sitting and lying, the angles relative to the gravitational direction of body parts are obtained using the signals of the accelerometers mentioned above. To calculate the angle changes of the body parts during dynamic posture i.e., walking, we provide angle changes in 
(a)

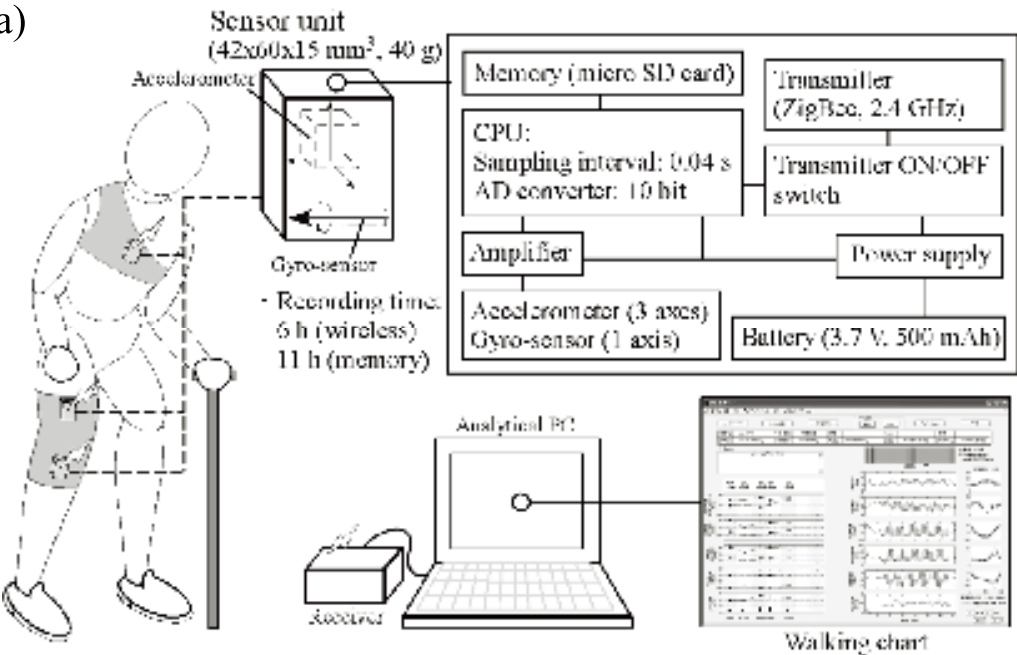

(b)

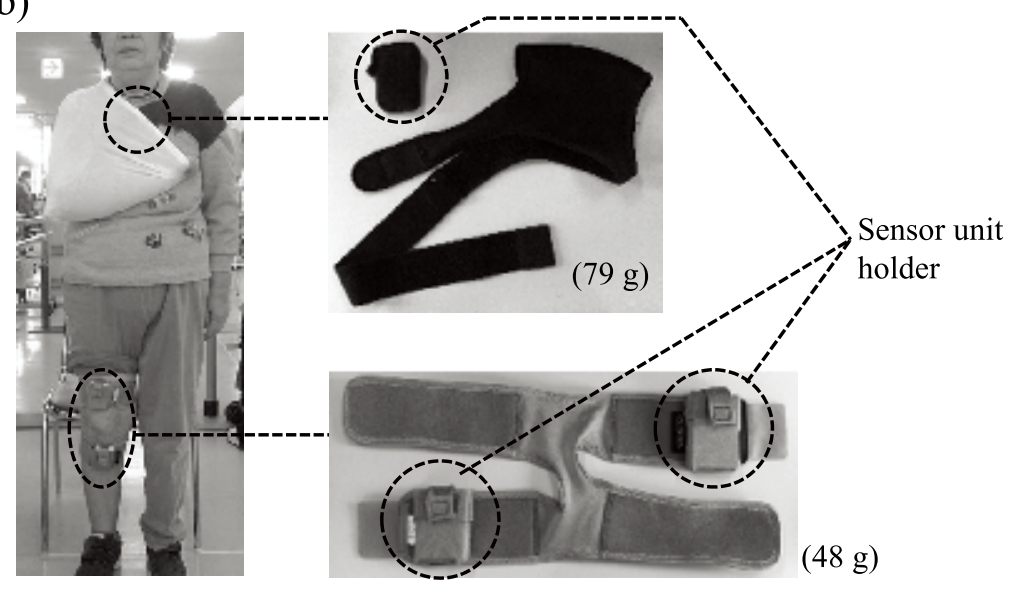

(c)

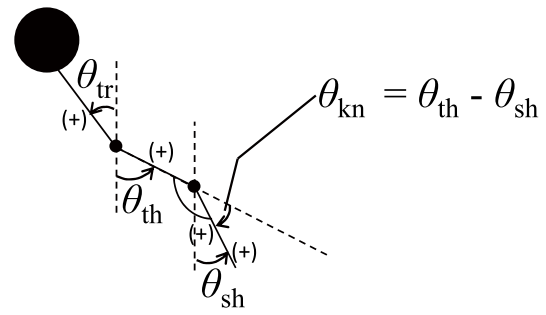

Fig. 1. Outline of wearable gait monitoring system for use in rehabilitation room (a). This system can be used to measure posture changes together with walking speed. Picture of subject's sensor attachment is also shown in the lower part (b). Definitions of trunk, thigh, and shank angles relative to the sagittal plane, and knee angle are also shown (c). 
high precision in which angular velocity outputs of the gyrosensors $(0.1-10 \mathrm{~Hz})$ are integrated. The initial angle values for integration are obtained from the sensor signals of the accelerometers in the static posture just before the walking. ${ }^{(20-22)}$ By repeating the determination of the initial angle values for every walking, accurate angle changes can be calculated without the effect of the sensor drift caused by the integration.

From these calculations, in this study, the angle changes of the trunk, thigh, and shank during walking, $\theta_{\mathrm{tr}}, \theta_{\mathrm{th}}, \theta_{\mathrm{sh}}$, respectively, as shown in Fig. 1(c), can be obtained accurately, for analyzing the posture changes and walking speed for the evaluation of the efficacy of rehabilitation.

On the other hand, the sensor units are attached to the subject's body using the shoulder-and knee-supporter-type sensor holders. The sensor holders (shoulder type: $79 \mathrm{~g}$, knee type: $48 \mathrm{~g}$ ) can be adjusted on the basis of the user's physical size and also enable close contact with the body. Also, when the patient is sitting on a chair at the start of measurement, the initial angle can be reset with $\theta_{\mathrm{tr}}=0 \mathrm{deg}, \theta_{\mathrm{th}}=90 \mathrm{deg}, \theta_{\mathrm{sh}}=0 \mathrm{deg}$, using software installed in a personal computer. Therefore, the system can accurately determine angle changes of the body segment throughout monitoring .

In Fig. 2, the method of measuring the walking speed is shown. In the previous system, the compass model using only angle changes of the thigh was used for the walking speed measurement. To improve this method, by using the two-link gait model based on the thigh and shank angle changes, the walking speed during the stance phase of the leg attached with the sensor units was calculated. The calculation step is as follows.

(1) The heel contact can be determined from the maximum value of the shank angle. In this point, the thigh and shank angle changes, $\theta_{\mathrm{thhc}}$ and $\theta_{\mathrm{sh}, \mathrm{c}}(\mathrm{deg})$, respectively, are obtained. The horizontal distance between the hip joint and heel, $D_{\mathrm{c}}(\mathrm{m})=L_{\mathrm{th}} \sin \theta_{\mathrm{th}, \mathrm{c}}$ $+L_{\mathrm{sh}} \sin \theta_{\mathrm{sh}, \mathrm{c}}$, was calculated using the subject's thigh and shank length, $L_{\mathrm{th}}$ and $L_{\mathrm{sh}}(\mathrm{m})$, respectively.

(2) The heel off can be determined from the minimum value of the thigh angle, for obtaining the thigh and shank angles, $\theta_{\mathrm{th}, \mathrm{o}}$ and $\theta_{\mathrm{sh}, \mathrm{o}}(\mathrm{deg})$, respectively. The horizontal distance between the hip joint and heel, $D_{\mathrm{o}}(\mathrm{m})=-L_{\mathrm{th}} \sin \theta_{\mathrm{th}, \mathrm{o}}-L_{\mathrm{sh}} \sin \theta_{\mathrm{sh}, \mathrm{o}}$, is calculated using the subject's leg length, $L_{\mathrm{th}}$ and $L_{\mathrm{sh}}(\mathrm{m})$.

(3) By summing $D_{\text {c }}$ and $D_{\mathrm{o}}$, the moving distance from heel contact to heel off, $D_{\text {co }}(\mathrm{m})$, is calculated.

(4) The period from the heel contact to heel off, $T_{\mathrm{co}}(\mathrm{s})$, is determined.

(5) The walking speed, $V_{\text {co }}(\mathrm{m} / \mathrm{s})$, is detected from $D_{\text {co }} / T_{\text {co }}$. The cadence, $C$ (steps $\left./ \mathrm{min}\right)$, was also calculated from the number of cycles during walking. By repeating the calculation of the values mentioned above for one walking cycle, the walking speed can be obtained continuously. In addition to these calculations, the values mentioned below are also obtained in order to evaluate the motion characteristics of the patient during walking.

(6) Knee angle, $\theta_{\mathrm{kn}}(\mathrm{deg})=\theta_{\mathrm{th}}-\theta_{\mathrm{sh}}$, as shown in Fig. 1(c), and variation width, $\theta_{\mathrm{kn}, \mathrm{w}}(\mathrm{deg})$, are determined.

(7) Trunk angles in the heel contact and heel off, $\theta_{\mathrm{tr}, \mathrm{c}}, \theta_{\mathrm{tr}, \mathrm{o}}(\mathrm{deg})$, respectively, are determined. 


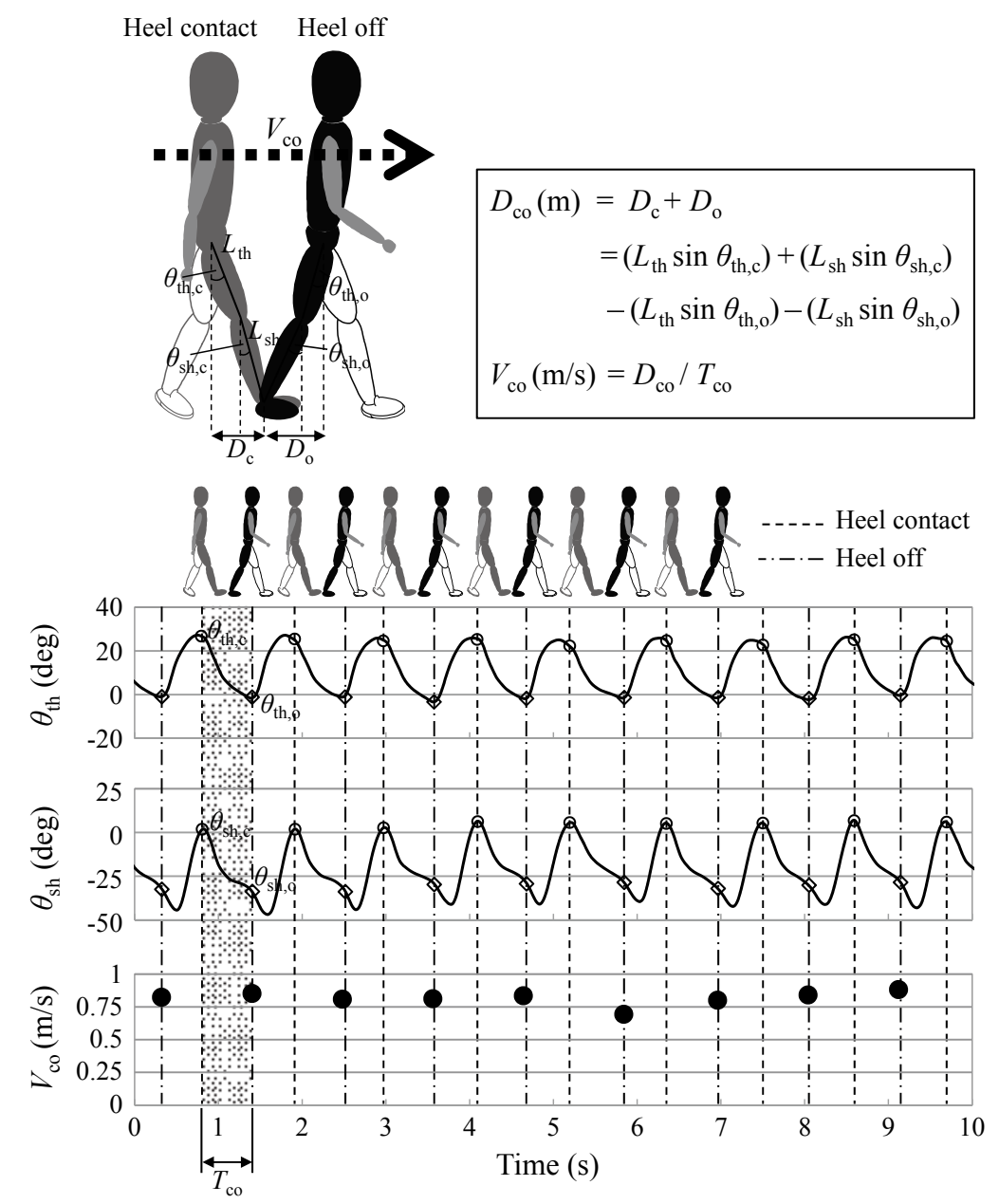

Fig. 2. Outline of analytical program for the patient's gait to obtain angles in heel contact and heel off and walking speed using a two-link gait model.

(8) Standard deviations, S.D., of the angle changes, $\theta_{\mathrm{tr}, \mathrm{sd}}, \theta_{\mathrm{th}, \mathrm{sd}}, \theta_{\mathrm{sh}, \mathrm{sd}}, \theta_{\mathrm{kn}, \mathrm{sd}}(\mathrm{deg})$, and those in the heel contact and heel off, $\theta_{\mathrm{tr}, \mathrm{c}, \mathrm{sd}}, \theta_{\mathrm{th}, \mathrm{c}, \mathrm{sd}}, \theta_{\mathrm{th}, \mathrm{s}, \mathrm{sd}}, \theta_{\mathrm{sh}, \mathrm{c}, \mathrm{sd}}, \theta_{\mathrm{sh}, \mathrm{o}, \mathrm{sd}}$, accumulated every walking cycle are determined.

In addition to these analyses, the posture changes of the patient can be visualized as a stick picture, making the PT easily understand the posture of the patient.

Figure 3(a) shows an experimental setup for evaluating the accuracy of the system in measuring the angle change and walking speed. Using a CCD camera, the movements of the markers attached to the subject's shoulder joint, hip (trochanter major), knee, and heel were simultaneously recorded at 30 frames per second. 
(a)

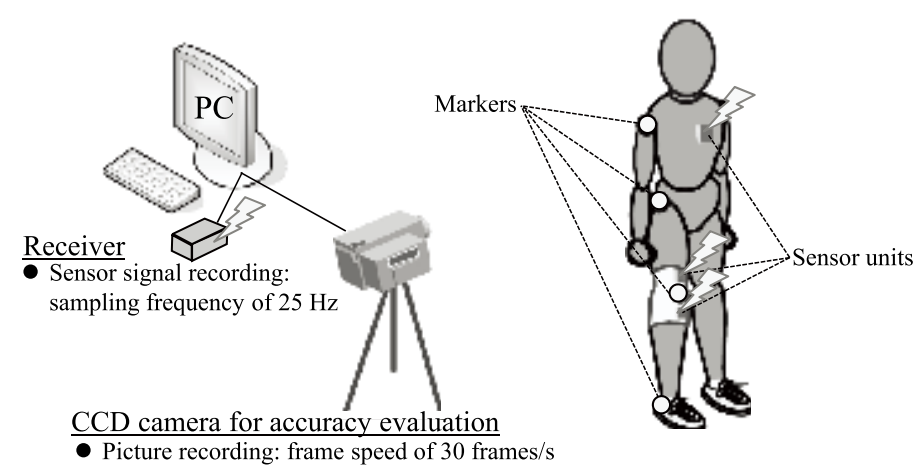

(b)

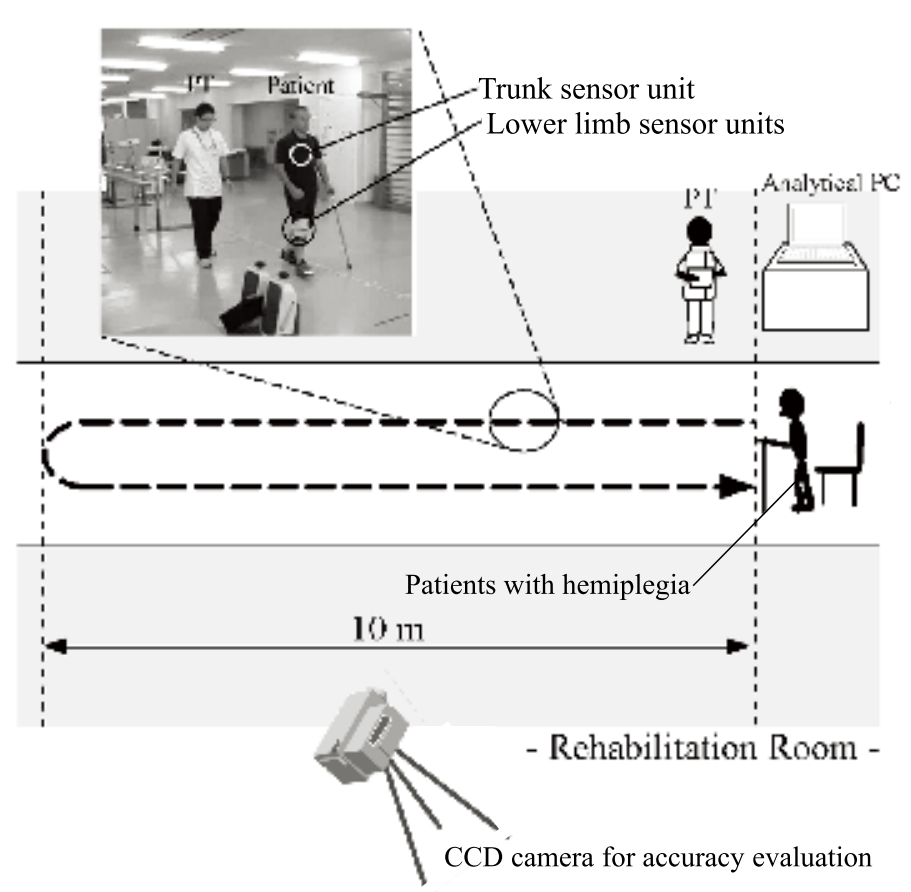

Fig. 3. Experimental setup for evaluating measurement accuracy in the angle change and walking speed (a). To investigate its applicability to rehabilitation, practical measurements during walking training in the patients with hemiplegia were carried out in the rehabilitation room (b).

Firstly, in front of the video camera, 7 healthy subjects (from 21 to 75 years) and 4 patients with hemiplegia (from 50 to 87 years) were asked to take several posture changes, e.g., walking, standing-up, sitting-up, etc. The actual angles relative to the 
gravitational direction were calculated using the position of the markers mentioned above. The angles estimated using the system were compared with the actual angle values to evaluate the accuracy of the angle change measurement.

On the other hand, 12 healthy subjects (from 21 to 75 years) and 10 patients (from 50 to 87 years) were asked to walk at various speeds. The actual walking speeds were calculated from the moving distance of the marker attached to the subject's trochanter major. The speeds estimated using the system were compared with the actual speeds to evaluate the accuracy of the walking speed measurement.

To investigate its applicability to the rehabilitation, we also carried out the practical monitoring of four patients with hemiplegia (from 50 to 69 years) during $10 \mathrm{~m}$ of walking training in the rehabilitation, as shown in Fig. 3(b). The signals from the sensors were recorded continuously throughout the rehabilitation. The lower limb sensors were attached on the paralyzed side, and the subjects used a cane (T-type or Q-type) during standing-up and walking.

Before the measurement, we obtained permission from the Ethical Review Board of the hospital and written informed consent was obtained from each of the patients.

\section{Results and Discussion}

Figure 4 shows typical recordings of the posture changes and walking speed in (a) a 24-year-old healthy male subject and (b) a 63-year-old male patient with hemiplegia (rehabilitation term: 62 days). In this figure, the angle changes relative to the sagittal plane of the trunk, thigh, and shank $\left(\theta_{\mathrm{tr}}, \theta_{\mathrm{th}}, \theta_{\mathrm{sh}}\right.$, respectively) are shown. The walking speeds from heel contact to heel off $\left(V_{\mathrm{co}}\right)$ are also plotted for every walking cycle. The angle changes determined from the CCD camera recordings are also shown in the graphs with an interval of the $0.33 \mathrm{~s}$. From these results, the angles estimated using the system coincided well with those determined from the CCD camera. Also, the system could be used to measure the walking speed continuously. In both healthy subject and patient, cyclic angle changes and stable walking speeds are observed. However, in the patient' $\mathrm{s}$ monitoring, the angle changes and walking speeds were smaller than those obtained in the healthy subject. Moreover, as shown in this figure, the system could measure the walking speed, such as not only the fast values of the healthy subject, but also the slow values of the patient with hemiplegia.

Figures 5(a)-5(c) show the scatter diagrams between the angles obtained from the sensor system and those from the CCD camera during posture changes in seven healthy subjects (from 21 to 75 years) and four patients with hemiplegia (from 50 to 87 years). From these results, fairly good linear relationships are observed in the trunk, thigh, and shank, demonstrating that the system could be used to measure the posture changes with high accuracy $(r=0.99)$.

Also, in Fig. 5(d), the accuracy of the walking speed measurement is shown in 12 healthy subjects (from 21 to 75 years) and 10 patients with hemiplegia (from 50 to 87 years). The horizontal axis shows the walking speeds calculated from the moving distance and periods using the CCD camera recordings. The vertical axis shows those calculated with the sensor system using the two-link gait model. As shown in this figure, 
(a)

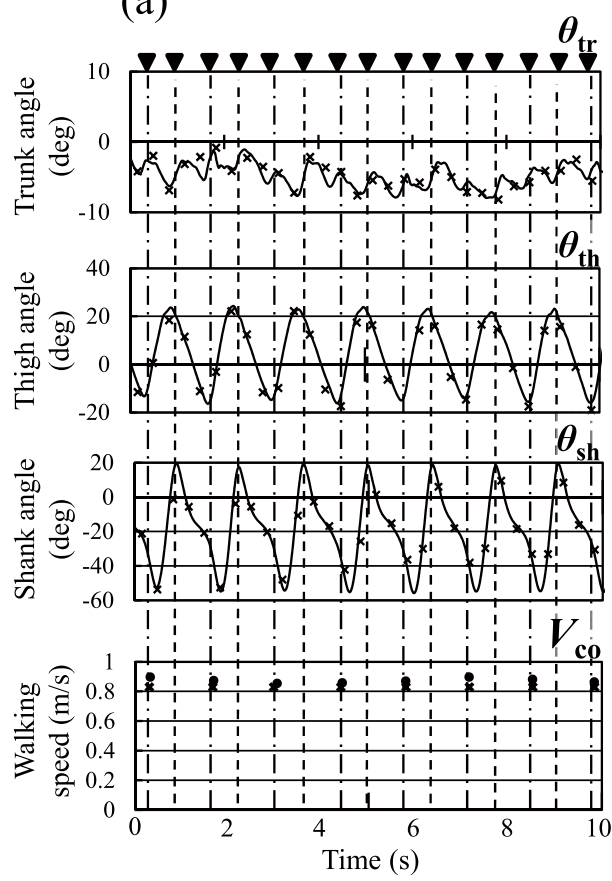

(b)

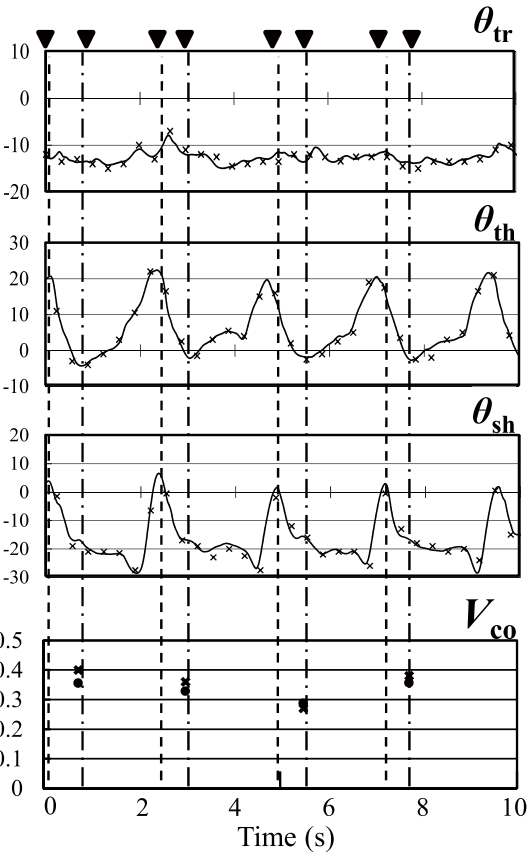

Heel contact and heel off obtained from sensor system

$\nabla$ Heel contact and heel off obtained from CCD

$X$ Values obtained using CCD with interval of $0.33 \mathrm{~s}$

Fig. 4. Results of the gait monitoring in (a) a 24-year-old healthy male subject and (b) 63-yearold male patient with hemiplegia (rehabilitation period: 62 days).

the values estimated using the sensor system coincided well with the actual values obtained with the CCD camera and a good linear relationship was obtained within a wide range of walking speeds. The results also demonstrated that the accuracy of this system ( $r$ $=0.99)$ is superior to that of the previous system $(r=0.96) .^{(19-22)}$ Moreover, a good linear relationship was also obtained in the patients with hemiplegia $(r=0.95)$.

As mentioned above, the system could be used to measure the posture changes and walking speed with high accuracy, and can be of practical use in the rehabilitation field. In the angular measurement, the good accuracy was obtained in a wide range of angles including walking motion. Thus, further investigations can be carried out, such as the analysis of activities including standing-up, sitting, and lying, and also the detailed motion characteristics of those postures. 
(a)

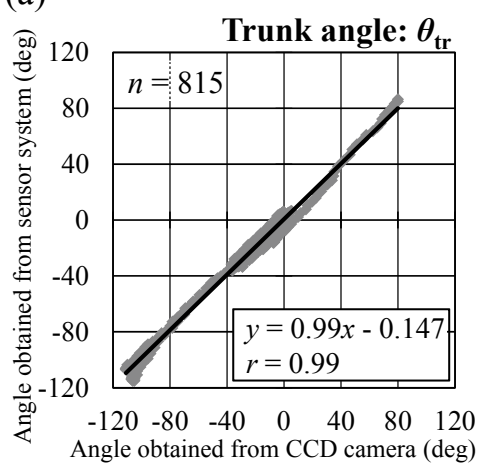

(c)

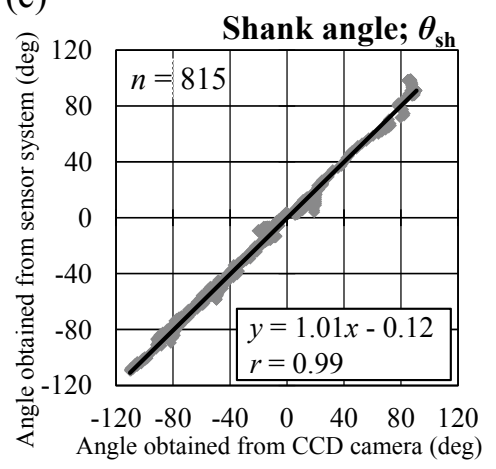

(b)

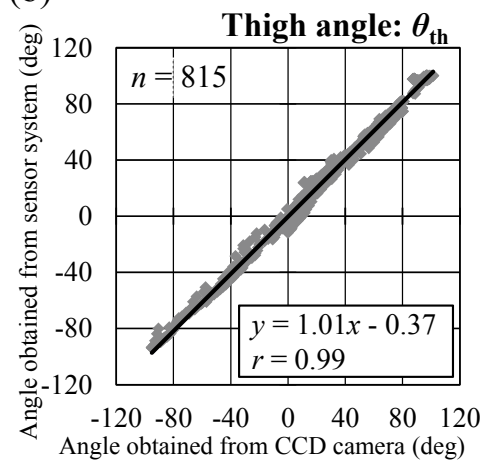

(d)

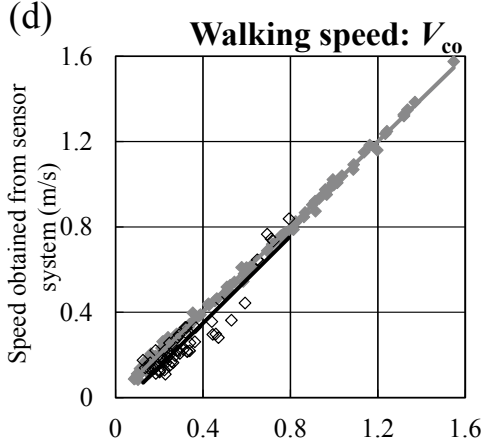

Speed obtained from CCD camera $(\mathrm{m} / \mathrm{s})$

Fig. 5. Correlation between values obtained from sensor system and those obtained from CCD cameras. Results in (a) trunk, (b) thigh, and (c) shank angles and (d) walking speed are shown.

Moreover, in the walking speed monitoring, good accuracy was obtained in a wide range of speeds. However, a slight underestimation was observed in the patients. In these cases, the patients were walking while swirling the leg, and thus, the angle changes relative to the sagittal plane of the thigh and shank were small. Therefore, walking speeds smaller than the actual values were detected. As future development, we will adapt a three-axes accelerometer and gyrosensor to the system and improve the method of calculating the walking speed.

Figure 6 shows practical recordings of the motion characteristics during walking training in the rehabilitation room. In this figure, the angle changes of the body segments and walking speed are shown. The right side of the angle changes shows averaged data 

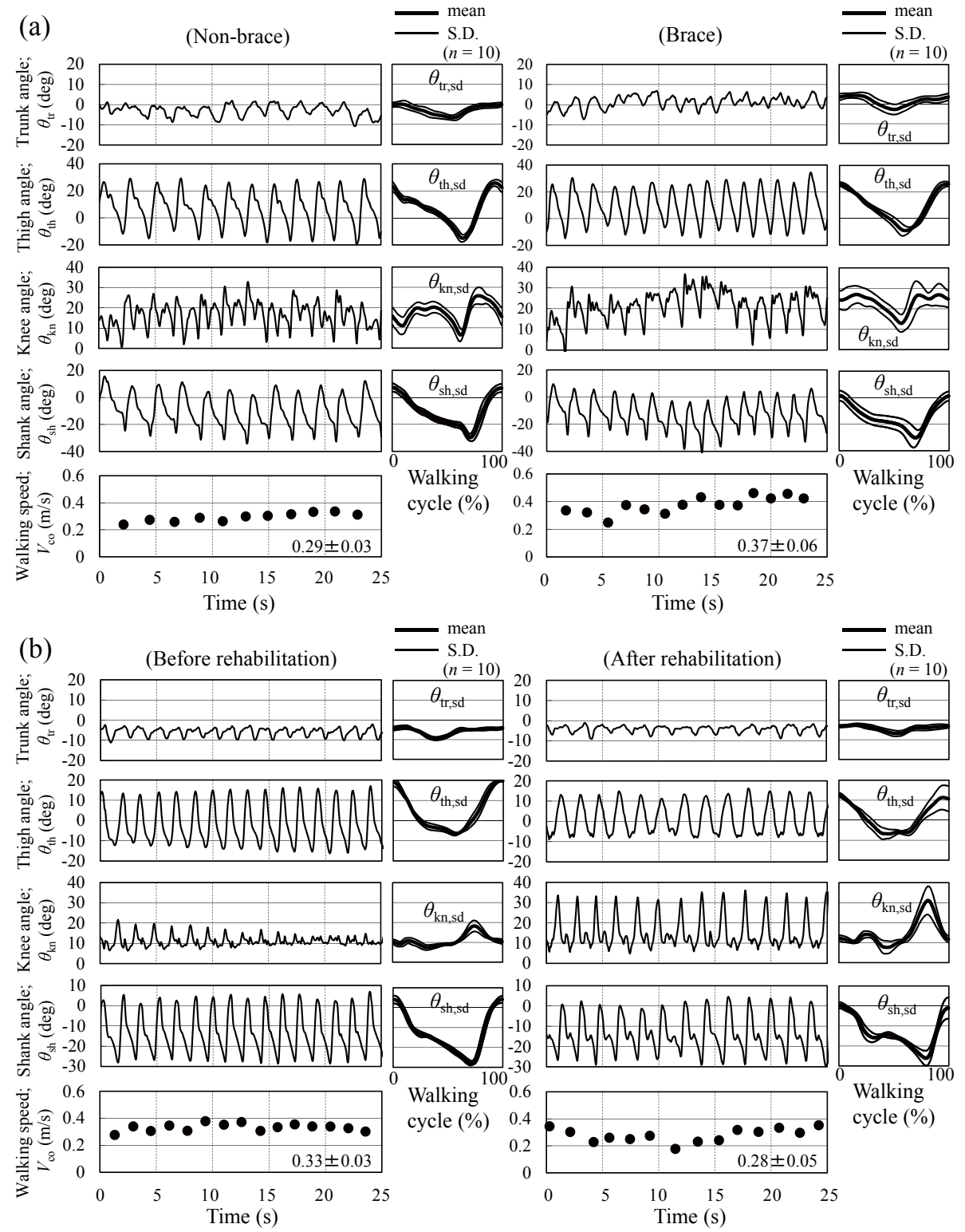

Fig. 6. Recordings of motion characteristics during walking training in rehabilitation room. In figure (a), motions in a male patient (69 years, rehabilitation: 880 days, hemiplegia: left side) with or without brace (MAFO) are shown. Also, figure (b) compares motion between before and after rehabilitation program in a female patient (62 years, rehabilitation: 21 days, hemiplegia: right side) attached with the brace. 
(accumulated 10 walking cycles) of the angles, represented as one walking cycle. The horizontal axis shows the walking cycle as a percentage from $0 \%$ (heel contact) to $100 \%$ (next heel contact). The bold lines denote the average and the thin lines denote \pm S.D. The average and S.D. values of the walking speed are also shown in the graph.

In Fig. 6(a), the motions of the male patient (69 years, rehabilitation: 880 days, hemiplegia: left side) with or without brace (MAFO) are shown. From these results, the subject could walk using the brace with the speed of $0.37 \mathrm{~m} / \mathrm{s}$ faster than those without the brace $(0.29 \mathrm{~m} / \mathrm{s})$. However, the decrease in the motion repeatability in the body segments was observed from the S.D. (maximum S.D. in $\theta_{\mathrm{kn}}= \pm 4.22 \mathrm{deg}$, nonbrace; \pm 7.34 deg, brace), showing the need of more walking training. Therefore, using these data, the PT could assess the efficacy and drawbacks of the brace attached to the subjects during the rehabilitation.

Also, Fig. 6(b) shows the comparison of the motion during walking between before and after the rehabilitation (about $30 \mathrm{~min}$ ) in the female patient (62 years, rehabilitation: 21 days, hemiplegia: right side) with the brace. In the rehabilitation, this patient had the stretch, massage, standing and walking training sessions, and so on. In the results, no fluctuations in the motion were seen before the rehabilitation, showing the high repeatability in the S.D. (maximum S.D. in $\theta_{\mathrm{kn}}= \pm 3.42 \mathrm{deg}$ ) of the angle changes. However, the range of the knee angle change was very small. After the rehabilitation, the range of the knee angle change became larger than those obtained before rehabilitation, while the repeatability of the angle changes decreases. These results show that the subject could control the gait by flexing and extending the knee; therefore, the effectiveness of the rehabilitation program could be observed. However, in the thigh, knee, and shank angle changes, the unstable fluctuations were observed in the periods of the extension phase of the hip and knee joints. Moreover, the decrease in the repeatability of the motion was also observed from the S.D. (maximum S.D. in $\theta_{\mathrm{kn}}= \pm 7.21$ deg) of the angle changes.

From these results, using the present system, the PT can easily understand the patient's posture changes during walking using the angular changes of the trunk, thigh, and shank. It is demonstrated through practical use that the system could measure the detailed changes in the motion characteristics with the walking training and the attachment of the brace, showing its usefulness as a quantitative assessment of the efficacy of the rehabilitation. Moreover, the motion characteristics that they cannot determine only by direct observation can be obtained quantitatively, and thus, the PT can feedback the motion drawbacks and improvement to the patients more accurately.

Figure 7 shows the long-term measurement of gait in (a) a 56-year-old male subject with hemiplegia of the right side and (b) a 50-year-old male subject with hemiplegia of the left side. These recordings show the walking speed $\left(V_{\mathrm{co}}\right)$, cadence $(C)$, range of the thigh angle change obtained using the difference between two angles in the heel contact and heel off $\left(\theta_{\mathrm{th}, \mathrm{c}}, \theta_{\mathrm{th}, \mathrm{o}}\right)$, and range of knee angle change during one walking cycle and S.D. (accumulated 20 walking cycles) of the thigh angle in heel contact. Each plot denotes the average value during $10 \mathrm{~m}$ of walking after rehabilitation.

From the results shown in Fig. 7(a), the walking speed $V_{\text {co }}$ and cadence $C$ became faster from 42 to 57 days and then the $V_{\text {co }}$ of about $0.6-0.7 \mathrm{~m} / \mathrm{s}$ and $C$ of about 110 steps/ 
(a)
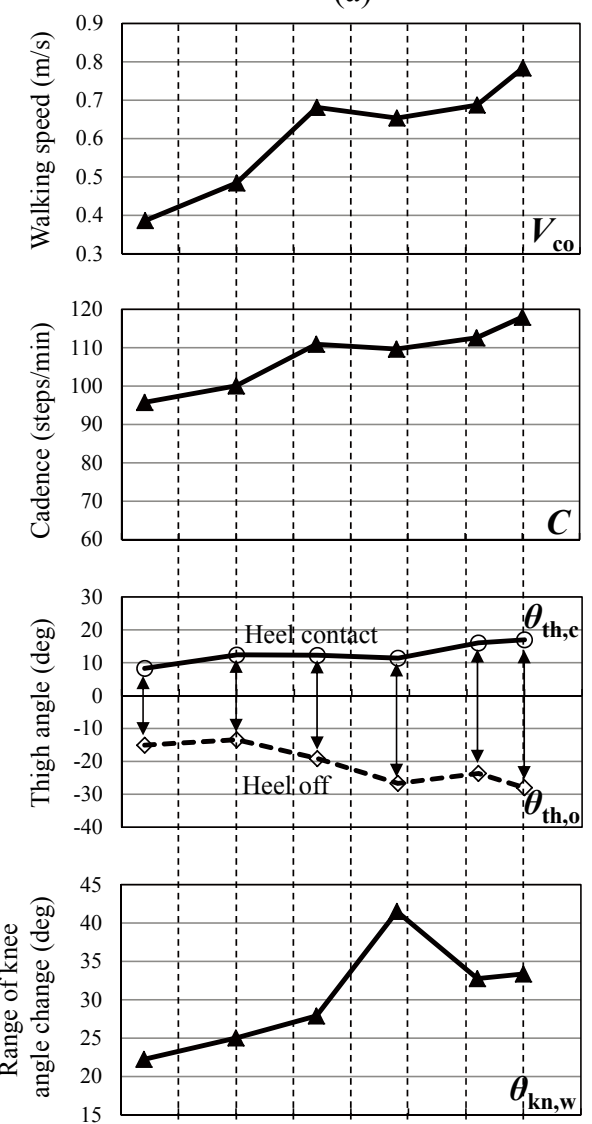

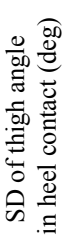

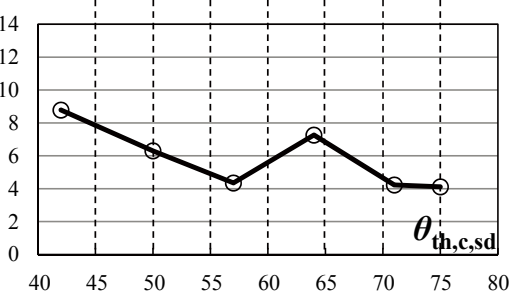

Rehabilitation length (day) (b)
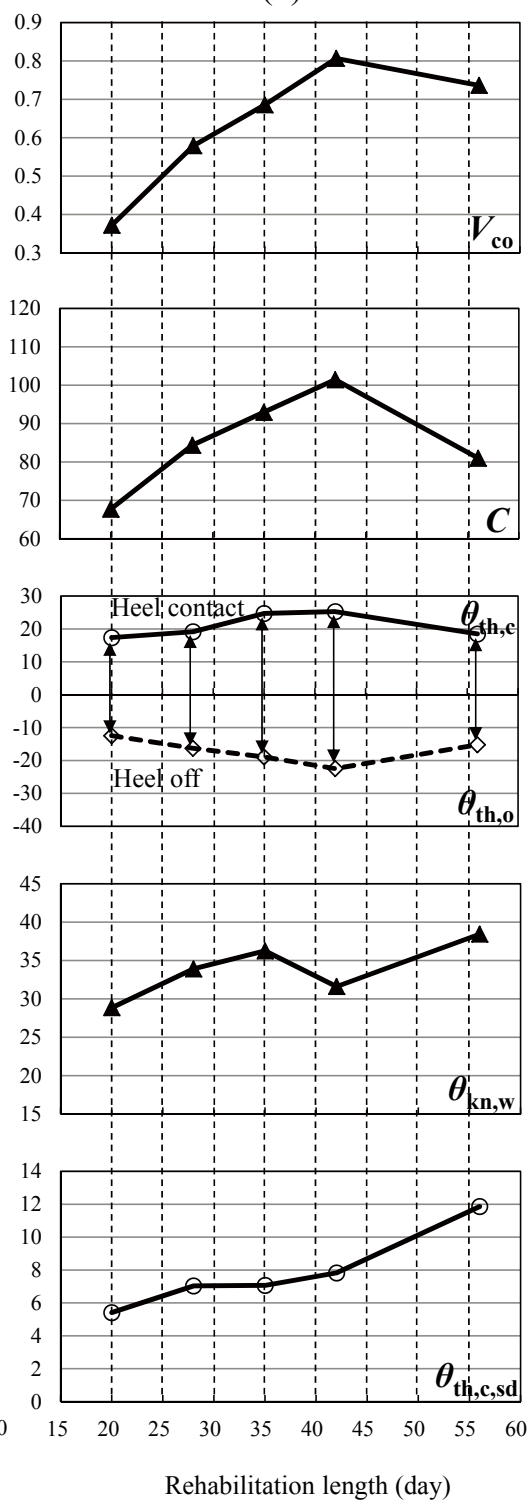

Fig. 7. Long-term measurement of gait in (a) a 56-year-old male subject with hemiplegia of the right side and (b) a 50-year-old male subject with hemiplegia of the left side. 
min were maintained. Therefore, performance and walking efficiency could be improved by the rehabilitation. Moreover, the ranges of the thigh and knee angle changes became larger; therefore, the joint motion was improved by the rehabilitation. On the other hand, the S.D. of the thigh angle in the heel contact, $\theta_{\text {th, c,sd }}$, i.e., the repeatability of thigh motion, became worse from 57 to 64 days while the walking speed, $V_{\text {co }}$, was maintained. However, after then, the S.D. values became small with increasing walking speed until 75 days, showing the improvement of the motion repeatability.

Also, as shown in Fig. 7(b), the walking speed $V_{\text {co }}$, cadence $C$, and angle changes became larger by 42 days. However, in 56 days, the walking speed $V_{\text {co }}$ and cadence $C$ became smaller than those obtained in 42 days and the $\theta_{\text {th,c,sd }}$ became larger, showing the decrease in the repeatability of the motion. Therefore, the patients would need further rehabilitation by the PT.

Therefore, the present system can be useful for the quantitative evaluation of the effectiveness of the long-term rehabilitation, setting up the final goal of the training, giving the permission for the walking without assistance by a therapist during daily living, and so on, using analysis results such as that shown in Fig. 7. As a future research, more measurements and analyses with a longer time and many subjects would be needed to investigate a more quantitative method of evaluating the efficacy of the rehabilitation. Also, the changes in the motion characteristics and recovery processes in many symptoms should be analyzed.

The PT may have to determine the rotation of the body segments and, thus, the angular measurements using the three-axes accelerometer and gyrosensor signals would also be needed. On the other hand, the sensor units of the lower limb were attached to only the subject's paralyzed side in this study. If the sensor units are attached to both sides, i.e., the paralyzed and healthy sides, a more detailed analysis can be carried out for the evaluation of the patient's motion, considering these viewpoints and improvements of the system as subjects for future investigation.

\section{Conclusions}

In this study, we developed a new gait monitoring system for use in the walking training in rehabilitation. The results demonstrated that the present system can be used to measure the posture changes, i.e., the trunk, thigh, and shank angle changes and the walking speed with good accuracy during walking. On the other hand, the system successfully functioned during rehabilitation in the hospital. It is clearly shown through practical monitoring that this system could be used to measure the detailed motion characteristics of the patients with hemiplegia as the changes in the trunk, thigh, and shank angles in the sagittal plane and walking speed for one walking cycle. We suggest that the efficacy of the rehabilitation can be quantitatively evaluated using the system.

Further investigations will be needed, such as the availability of measurements for a longer period of time in more patients, three-dimensional analysis of the motion during walking, and application for analysis of other motions including standing-up, sitting, lying, and so on. 


\section{Acknowledgement}

The authors wish to thank the patients and therapists in the rehabilitation room of Fujimoto Hayasuzu Hospital. This work was supported in part by the Japanese Ministry of Internal Affairs and Communications under the Strategic Information and Communications R\&D Promotion Programme (SCOPE; No. 102305004, 2010 and 2011), for which we wish to express our sincere appreciation.

\section{References}

1 A. Hendry, W. Gilchrist, G. Duncan, A. L. Evans and D. C. Smith: Med. Biol. Eng. Comput. 28 (1990) 602.

2 J. O. Brooks, L. Friedman, D. L. Bliwise and J. A. Yesavage: Sleep 16 (1993) 151.

3 B. D. Evans and A. E. Rogers: Appl. Nurs. Res. 7 (1994) 75.

4 T. Tamura, M. Sekine, M. Ogawa, T. Togawa and Y. Fukui: Methods Inf. Med. 36 (1997) 356.

5 M. Sekine, T. Tamura, M. Akay, T. Fujimoto, T. Togawa and Y. Fukui: IEEE Trans. Rehabil. Eng. 10 (2002) 188.

6 M. Makikawa and H. Izumi: Proc. MedInfo 95 (1995) 747.

7 P. H. Veltink, B. J. Bussmann, D. Vries, L. J. Martens and R. C. V. Lummel: IEEE Trans. Rehabil. Eng. 4 (1996) 375.

8 J. Fahrenberg, F. Foerster, M. Smeja and W. Muller: Psychophysiology 34 (1997) 607.

9 B. Najafi, K. Aminian, A. Paraschiv-Ionescu, F. Loew, C. J. Bula and P. Robert: IEEE Trans. Biomed. Eng. 50 (2003) 711.

10 R. Moe-Nilssen: Arch. Phys. Med. Rehabil. 79 (1998) 1377.

11 R. E. Mayagoitia, J. C. Lotters, P. H. Veltink and H. Hermens: Gait and Posture 16 (2002) 55.

12 H. Zhu, G. F. Harris, J. J. Wertsch, W. J. Tompkins and J. G. Webster: IEEE Trans. Biomed. Eng. 38 (1991) 710.

13 S. Miyazaki: IEEE Trans. Biomed. Eng. 44 (1997) 753.

14 K. Tong and M. H. Granat: Med. Eng. Phys. 21 (1999) 87.

15 K. Aminian, B. Najafi, C. Bula, P. F. Leyvraz and Ph. Robert: J. Biomech. 35 (2002) 689.

16 R. Williamson and B. J. Andrews: Med. Biol. Eng. Comput. 39 (2001) 1.

17 H. J. Luinge and P. H. Veltin: Med. Biol. Eng. Comput. 43 (2005) 273.

18 H. Dejnabadi, B. M. Jolles, E. Casanova, P. Fua and K. Aminian: IEEE Trans. Biomed. Eng. 53 (2006) 1385.

19 S. Tanaka, K. Yamakoshi and P. Rolfe: Med. Biol. Eng. Comput. 32 (1994) 357.

20 K. Motoi, S. Tanaka, M. Nogawa and K. Yamakoshi: Proc. SICE Annu. Conf. (2003) 563.

21 K. Motoi, S. Tanaka, M. Nogawa and K. Yamakoshi: Trans. Jpn. Med. Biol. Eng. 41 (2003) 35 .

22 K. Motoi, S. Tanaka, Y. Kuwae, T. Yuji, Y. Higashi, T. Fujimoto and K. Yamakoshi: J. Rob. Mechatron. 19 (2007) 656. 